\title{
Distribution and preliminary conservation assessments of commonly used forest species in the Nepalese Himalayas
}

\author{
B. Adhikari ${ }^{*}$, C. A. Pendry ${ }^{1}$, I. E. Måren ${ }^{2}$, K. R. Bhattarai ${ }^{3,4}$ and R. P. Chaudhary \\ Balancing the trade-offs between biodiversity conservation and ecosystem service \\ delivery is a colossal challenge in the areas of the globe with high productivity \\ and high demand, such as in south Asia. In order to meet this challenge, we need \\ enhanced knowledge of the species constituting these semi-natural systems. This \\ paper reports the country-level preliminary conservation assessments for 153 woody \\ plant species from the Middle Hills in central Nepal based on the IUCN criteria. \\ Distribution maps and threat categories are provided for all species. Ten species \\ are categorized as Near Threatened, two as Endangered and one as Vulnerable. \\ Conservation assessments could not be completed for 24 species because of \\ insufficient distribution data.
}

Key words: Conservation assessments, forest species, Himalayas, species distribution

I

$\mathrm{n}$ the global north, it is widely recognised that the conservation of semi-natural landscapes and their associated species are of paramount importance for the conservation of biodiversity, but these landscapes do not receive the same recognition in the global south. In south Asia, traditional semi-natural landscapes are still the backdrop for rural livelihoods, and cover large land areas. However, many of the region's traditional land uses are changing due to agricultural intensification or abandonment caused by socioeconomic change (Sharma, 2016) in these tightlylinked social-ecological systems. Enhanced knowledge of the dynamics between land use and biodiversity will be critical for future successful biodiversity conservation and ecosystem service delivery. The shift towards a system's view where humans are seen as part of the system (Berkes, 2004; Folke, 2006; Sharma, 2016) will benefit both biodiversity conservation and ecosystem service delivery in Nepal and other countries in the region. A recent study on species diversity, forest structure, ecosystem services and forest management practices both in the community forests (CFs) and government managed forests (GMFs) at Panchase, situated towards the west of Pokhara (Måren et al., 2013) found that the CFs had greater species diversity and less degradation than the GMFs, which in practice acts as a resource which is open for unrestricted exploitation by all. The community forest user groups (CFUGs) at Panchase manage their forests so as to improve their condition by removing undesirable species in favour of the growth of the species with high value for fuel, fodder, fibre and medicine. However, it is not clear whether greater species diversity has any relationship with the numbers of rare species growing in the forest. Nepal's flora is believed to comprise around 7,000 species of flowering plants (Press et al., 2000; Watson et al., 2011; Miehe et al., 2015), but only few of its species have been evaluated for their conservation status. The distribution data which are used to generate conservation assessments is derived primarily from herbarium specimens (Rich and Lewis, 1999; Antonovics et al., 2003), but these collections are very unevenly spread across Nepal (Watson et al., 2011), so the distribution patterns of most species are inadequately known.

In this study, we examined six locations in central Nepal, the three of which are within the Protected Areas (National Parks or Conservation Areas) and the rest three are outside the Protected Areas, in order to further examine the effects of different legal frameworks on maintenance of forest biodiversity. This paper reports the preliminary conservation assessments for all the species

\footnotetext{
Royal Botanic Garden Edinburgh, 20a Inverlieth Row, Edinburgh, EH3 5LR, Scotland UK. *E-mail: B.Adhikari@rbge.ac.uk

Arboretum and Botanical Gardens, the University Museum, University of Bergen, Allegt. 41, 5006 Bergen, Norway

Sheridan Davis Campus, 7899 McLaughlin Rd, Brampton, ON L6Y 5H9, Canada

Himalayan Resource \& Development Center, GPO Box 7426, Kathmandu, Nepal

Research Centre for Applied Science and Technology (RECAST), Tribhuvan University, Kirtipur, Kathmandu, Nepal
} 
found in this study.

\section{Materials and methods}

\section{Study sites}

Three forested areas in central Nepal are studied, each with a study site within the protected area (National Park or Conservation Area) and an equivalent study site outside the protected area. These areas were Annapurna (Ghorepani inside the Annapurna Conservation Area and Panchase outside the protected area), the Kathmandu Valley (Shivapuri-Nagarjun National Park inside and Chandragiri outside the protected area) and Langtang (Langtang National Park inside and Bhalche outside the protected area) (Fig. 1). The study was conducted in the pre-monsoon season from February to June, 2010. Both the CFs and the GMFs (excluding plantations) were sampled using stratified random sampling. Sample plots of $10 \mathrm{~m} \times 10 \mathrm{~m}$ size were laid out across the study sites with similar the biophysical factors and elevation (Måren et al. 2013 for further details). In each study site, equal numbers of plots (180) were sampled, totalling 540 plots in the three regions (six sites). Results from $\mathrm{pH}$ and loss on ignition (LOI) analyses indicated only small differences in the soil conditions of the sites and the regions.

In the mid-hills, Oak-Laurel forests are situated at higher elevations while the mixed SchimaCastanopsis forests are found at lower elevations. These forests differ considerably in their floristic composition and ecology (Dobremez, 1976). Quercus semecarpifolia $\mathrm{Sm}$. is the dominant tree species in the Oak-Laurel forests with the species of laurel such as Lindera pulcherrima (Nees) Hook. f., Neolitsea pallens (D. Don) Momiy. \& H. Hara ex H. Hara, Machilus duthiei King ex Hook. f. and M. odoratissima Nees. The SchimaCastanopsis forests are dominated by Schima wallichii (DC.) Korth., Castanopsis indica (Roxb.) Miq. and C. tribuloides (Sm.) A.DC.

Other species which are also commonly found in the mid-hill forests include several species of Rhododendron, Acer spp., Prunus spp., Quercus glauca Thunb., Quercus lamellosa Sm., Quercus lanata Sm., Lyonia ovalifolia (Wall.) Drude, Eurya acuminate DC., Ilex dipyrena Wall., Symplocos ramosissima Wall. ex G. Don and Daphniphylum himalense (Benth.) Mull. Arg.
Pinus wallichiana A. B. Jacks. is found at the higher elevations while $P$. roxburghii Sarg. is noticed at the lower altitudes.

The less commonly occurring species include Magnolia doltsopa (Buch.-Ham. ex DC.) Figlar, Taxus wallichiana Zucc., Edgeworthia gardneri (Wall.) Meisn. etc. These forests are home to a number of important species of wildlife such as Himalayan black bear (Ursus thibetanus), tiger (Panthera tigris), Indian muntjak (Muntiacus muntjak), common leopard (Panthera pardus), jackal (Canis aureus) and several species of bats (Aryal and Dhungel, 2009; Miehe et al., 2015).

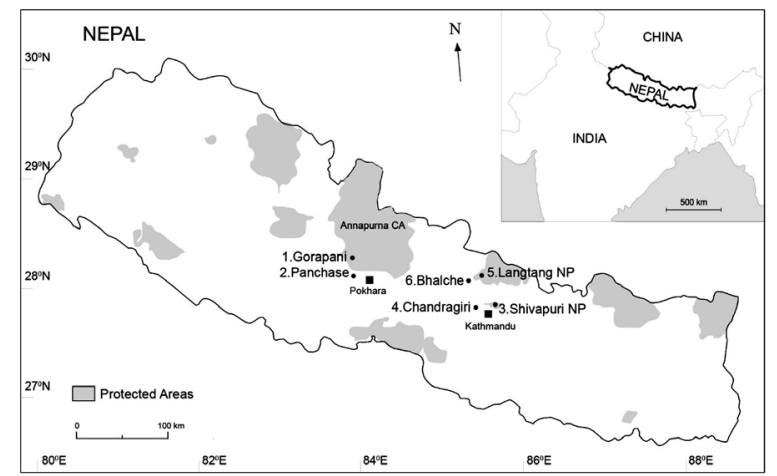

Fig. 1: Map showing the localities of the six study sites within the three regions in central Nepal, the Himalayas

\section{Calculation of conservation assessments}

The herbaria at the Royal Botanic Garden Edinburgh Herbarium (E), the Natural History Museum London (BM) and the National Herbarium and Plant Laboratories, Kathmandu (KATH) were consulted for specimens of the 153 woody species recorded during the study. All the specimens were photographed and data-based in the 'Padme database', which is used to manage all information for the Flora of Nepal project. Altogether, 4,374 herbarium specimens $(1,927$ specimens recorded from the $\mathrm{E}, 1,239$ specimens from the BM and 1,208 from the KATH) together with the occurrence-records in the present study and unvouchered field records of the occurrences of the unambiguously identified common species in the 'Padme database' were used for the assessments. In addition to this, the distributions of species in the neighbour countries were also taken into consideration while assigning the categories. The assessments were based mostly on criteria B and A of the IUCN Categories and the criteria using extent of occurrence (EOO) 
and area of occupancy (AOO) plus evidence (or inferring) of decline in the habitat (IUCN, 2016). Species which are very close to qualifying or likely to qualify for a threatened category (critically endangered, endangered or vulnerable) in the near future were categorized as Near Threatened (NT). Evidence of population size and/or reduction was, generally, not available. The EOO was calculated using GeoCAT (Bachman et al. 2011; http://geocat.kew.org/) while the AOO using a facility in the 'Padme database

\section{Results and discussion}

One hundred and fifty-three woody plant species were recorded from the six study sites, comprising 80 species of trees, 48 shrubs and 25 woody climbers (Annex I). The highest species richness of trees and climbers were recorded at the Annapurna sites (trees 53; climbers 21), followed by the Kathmandu Valley (trees 44; climbers 18) and the Langtang sites (trees 26; climbers 11). There was a greater diversity of shrubs in the Kathmandu sites (30) and the Annapurna sites (30) followed by the Langtang sites (16). The most commonly recorded family was Rosaceae (26 species) followed by Lauraceae (9 species), Fagaceae and Ericaceae (both 7 species).

\section{Preliminary conservation assessment}

Two species, Taxus wallichiana Zucc. and Hoya edenii King ex Hook. f. were categorised as 'Endangered' (EN), one species, Hypericum cordifolium Choisy as 'Vulnerable' (VU), ten species as 'Near Threatened' (NT) and 116 species as 'Least Concern' (LS). There were insufficient data to calculate conservation assessments for 24 species, and so those species were categorised as 'Data Deficient' (DD) (Table 1). The complete list of the species (trees, shrubs and climbers) found in the study sites along with their preliminary conservation assessments is presented in Annex I.

Tree species: Among the 80 species of trees, seven were categorised as 'Near Threatened' (NT) based on the IUCN criteria; the species being Abies spectabilis (D. Don) Mirb., Acer caudatum Wall., Aesculus indica (Colebr. ex Cambess.) Hook., Camellia kissi Wall., Eriobotrya elliptica Lindl., Euonymus pendulus Wall. and Litsea doshia (D. Don) Kosterm. T. wallichiana is the only tree species listed as 'EN' (A2 a, c, d). The population of this species is decreasing at an alarming rate because of commercial demand (Liu et al., 2011; Poudel et al., 2012; Gajurel et al., 2013), and it has been listed in the CITES Appendix 2 since 1995. Fifty eight species were fairly well distributed, and were categorized as 'LC' while 14 species were placed under 'DD'.

Shrubs: One species, H. cordifolium Choisy was recorded as 'VU' (B1 a, b). Three species, D. bholua Buch.-Ham. ex D. Don, D. papyracea Wall. ex Steud. and Edgeworthia gardneri (Wall.) Meisn. were recorded as 'NT' while thirty-seven species fell into 'LC' category, and seven were categorized as 'DD'.

Climbers: One species, Hoya edenii King ex Hook. f. was assessed as 'EN', (B1 a, b) while 21 were recorded as 'LC', and three were categorized as 'DD'.

\section{Conclusion}

This study clearly reflects the limitations of the data which are currently available. Almost 15\% of the species were classified as 'Data Deficient' as their distributions were too poorly known to confidently assign them to any category. Several of these species have very limited distributions, and are known only from a few specimens, and so it is quite possible that a significant number of them are actually under threat of depletion or extinction. Looking at the maps of some of the common species, such as $P$. roxburghii and $P$. Wallichiana (Annex II), it is evident that these species are certainly under-recorded and the data set is insufficient to make accurate conservation assessments for these species. Clearly, more distribution records are needed before we can

Table 1: Preliminary conservation assessments based on the IUCN criteria for 153 woody plant species recorded at the Ghorepani, Panchase, Shivapuri, Chandragiri, Langtang and Bhalche sites in central Nepal

\begin{tabular}{cccccc}
\hline $\begin{array}{c}\text { Data } \\
\text { Deficient } \\
\text { (DD) }\end{array}$ & $\begin{array}{c}\text { Least } \\
\text { Concern } \\
\text { (LC) }\end{array}$ & $\begin{array}{c}\text { Near } \\
\text { Threatened } \\
(\mathbf{N T})\end{array}$ & $\begin{array}{c}\text { Vulnerable } \\
\text { (VU) }\end{array}$ & $\begin{array}{c}\text { Endangered } \\
(\text { EN) }\end{array}$ & $\begin{array}{c}\text { Critically } \\
\text { Endangered } \\
\text { (CE) }\end{array}$ \\
\hline 24 & 116 & 10 & 1 & 2 & 0 \\
\hline
\end{tabular}


be certain of the conservation status of these commonly occurring and widely utilized Nepalese woody plants.

Many natural resource systems, here exemplified by forests, fall under collective management or are subject to use by multiple individuals, often for a variety of purposes (Poteete and Ostrom, 2004). Sustaining these resources in the face of economic and demographic pressures depends upon an array of interdependent components including legislation and local engagement. In order to facilitate evidence-based natural resource management, we need to enhance our knowledge regarding the species richness, composition and dynamics of these systems. Pandey (2007) found comparatively higher species richness in the community forests than in the national parks and government forests he investigated, and in the sacred groves of the Western Ghats of India. Bhagwat et al. (2005) found informal protection traditions to contribute to successful biodiversity conservation. We see similar trends in some of our material; however, we cannot see this as an overriding trend for the data set as a whole. In other words, these dynamics within social-ecological systems are context-dependent which call for enhanced knowledge in order to manage both ecosystem service delivery to the local people, and contribute to biodiversity conservation.

\section{Acknowledgements}

We would like to thank Mr. Bishnu Chapagain, Mr. Ashok Chaudhary, Mr. Kuber Bhatta, Mr. Rupesh Gurung, Ms. Lila Sharma, Ms. Asha Suwal, Mr. Rajesh Shrestha and Ms. Keith McInturff for their assistance in our fieldwork. We are thankful to all the villagers who helped us in our fieldwork. We acknowledge Machhapuchhre Development Organisation (MDO) and Ms. Bashuda Gurung for providing insightful information on the Panchase area. We are grateful to the Norwegian Research Council (190153/ V10) and Grolle Olsens Legat for their financial support to accomplish this study.

\section{References}

Antonovics, J., Hood, M. E., Thrall, P. H., Abrams, J. Y. and Duthie, G. M. 2003. Herbarium studies on the distribution of anther-smut fungus (Microbotryum violaceum) and Silene species (Caryophyllaceae) in the eastern United States. American Journal of Botany 90: 1522-1531. doi:10.3732/ajb.90.10.1522 accessed on 14 June, 2016.

Aryal, A. and Dhungel, S. K. 2009. Species diversity and distribution of bats in Panchase region of Nepal. Tigerpaper 36 (2): 14-18.

Bachman, S., Moat, J., Hill, A. W., de la Torre, J. and Scott, B. 2011. Supporting Red List threat assessments with GeoCAT: geospatial conservation assessment tool. ZooKeys 150: 117-126. http://dx.doi.org/ 10.3897/ zookeys.150.2109 accessed on 14 June, 2016.

Berkes, F. 2004. Rethinking community-based conservation. Conservation Biology 18 (3): 621-630.

Bhagwat, S. A., Kushalappa, C. G., Williams, P. H. and Brown, N. D. 2005. The role of informal protected areas in maintaining biodiversity in the Western Ghats of India. Ecology and Society 10: 8.

Dobremez, J. F. 1976. Le Nepal: Ecologieet Biogeographie. Centre National de la Recherche Scientifique, Paris, France.

Folke, C. 2006. Resilience: The emergence of a perspective forsocial-ecological systems analyses. Global Environmental Change 16: 253-267.

Gajurel, J. P., Cornejo, C., Werth, S., Shrestha, K. K. and Scheidegger, C. 2013. Development and characterization of microsatellite loci in the endangered species Taxus wallichiana (Taxaceae). Applications in Plant Sciences 1 (3): 1200281. http://doi.org/10.3732/apps. 1200281 accessed on 18 July, 2016.

IUCN. 2016. Guidelines for Using the IUCN Red List Categories and Criteria, Version 12. Prepared by the Standards and Petitions Subcommittee, The International Union for Conservation of Nature. http://www.iucnredlist.org/documents/ RedListGuidelines.pdf accessed on 14 December, 2016.

Liu J., Gao, L. M., Li, D. Z., Zhang, D. Q. and Moller, M. 2011. Cross-species amplification and development of new microsatellite loci 
for Taxus wallichiana (Taxaceae). American Journal of Botany 98: e70-e73.

Måren, I., Bhattarai, K. R. and Chaudhary, R. P. 2013. Forest Ecosystem Services and Biodiversity in Contrasting Himalayan Forest Management Systems Environmental Conservation. Published online doi:10.1017/ S0376892913000258 accessed on 15 October, 2016.

Miehe, G., Pendry, C. A. and Chaudhary, R. 2015. Nepal: An Introduction to the Natural History, Ecology, and Human Environment of the Himalayas. Royal Botanic Garden Edinburgh, Edinburgh, UK.

Pandey, S. S. 2007. Tree Species Diversity in Existing Community-based Forest Management Systems in Central Mid-hills of Nepal. Swedish Biodiversity Centre, Swedish University of Agricultural Sciences, Uppsala, Sweden.

Poteete, A. R. and Ostrom, E. 2004. Heterogeneity, Group Size and Collective Action: The Role of Institutions in Forest Management. Development and Change 35: 435-461. doi:10.1111/j.1467-7660.2004.00360.x accessed on 9 January, 2017.
Poudel, R. C., Möller, M., Gao, L. M., Ahrends, A., Baral, S. R., Liu, J., Thomas, P. and Li, D. Z. 2012. Using Morphological, Molecular and Climatic Data to Delimitate Yews along the Hindu Kush-Himalaya and Adjacent Regions. PLoS ONE 7 (10): e46873. doi: 10.1371/journal.pone.0046873 accessed on 16 December, 2016.

Press, J. R., Shrestha, K. K. and Sutton, D. A. 2000. Annotated Checklist of the Flowering Plants of Nepal. The Natural History Museum, London, UK.

Rich, T. C. G. and Lewis, J. 1999. Use of herbarium material for mapping the distribution of Erophila (Brassicaceae) taxa sensu Filfilan and Elkington in Britain and Ireland. Watsonia 22: 377-385.

Sharma, L. N. 2016. The Disturbance-Diversity Relationship: Integrating Biodiversity Conservation and Resource Management in Anthropogenic Landscapes. PhD Thesis, Department of Biology, University of Bergen, Norway.

Watson, M. F., Akiyama, S., Ikedo, H., Pendry, C., Rajbhandari, K. R. and Shrestha, K. K. 2011. Flora of Nepal (vol. 3). The Royal Botanical Garden Edinburgh, Edinburgh, UK. 
Annex I: List of the species recorded in the Annapurna (A) Region (Panchase and Ghorepani), the Kathmandu (K) Valley Region (Chandragiri and Shivapuri) and the Langtang (L) Region (Bhalche and Langtang], and their preliminary conservation assessment

\begin{tabular}{|c|c|c|c|c|c|c|}
\hline \multirow{2}{*}{ S.N. } & \multirow{2}{*}{ Scientific name } & \multirow{2}{*}{ Family } & \multicolumn{3}{|c|}{ Recorded from } & \multirow{2}{*}{ P. Con. Asses. } \\
\hline & & & $\mathbf{A}$ & $\mathbf{K}$ & $\mathbf{L}$ & \\
\hline \multicolumn{7}{|c|}{ TREES } \\
\hline 1 & Abies spectabilis (D.Don) Mirb & Pinaceae & $\checkmark$ & & & Near Threatened (NT) \\
\hline 2 & Acer caesium Wall. ex Brandis & Sapindaceae & $\checkmark$ & & & Data Deficient (DD) \\
\hline 3 & Acer campbellii Hook. f. \& Thomson ex Hiern & Sapindaceae & & & $\checkmark$ & Least Concern (LC) \\
\hline 4 & Acer caudatum Wall. & Sapindaceae & $\checkmark$ & & & Near Threatened (NT) \\
\hline 5 & Acer sterculiaceum Wall. & Sapindaceae & $\checkmark$ & & & Least Concern (LC) \\
\hline 6 & Actinodaphne angustifolia Nees & Lauraceae & $\checkmark$ & & & Data Deficient (DD) \\
\hline 7 & Actinodaphne sikkimensis Meisn. & Lauraceae & $\checkmark$ & & & Data Deficient (DD) \\
\hline 8 & Aesculus indica (Colebr. ex Cambess.) Hook & Sapindaceae & $\checkmark$ & & & Near Threatened (NT) \\
\hline 9 & Alnus nepalensis D.Don & Betulaceae & $\checkmark$ & & & Least Concern (LC) \\
\hline 10 & Benthamidia capitata (Wall.) H. Hara & Cornaceae & & & $\checkmark$ & Least Concern (LC) \\
\hline 11 & Betula alnoides Buch.-Ham. ex D.Don & Betulaceae & $\checkmark$ & $\checkmark$ & $\checkmark$ & Data Deficient (DD) \\
\hline 12 & Camellia kissi Wall. & Theaceae & $\checkmark$ & $\checkmark$ & & Near Threatened (NT) \\
\hline 13 & Carpinus viminea Lindl. & Betulaceae & & $\checkmark$ & & Least Concern (LC) \\
\hline 14 & Castanopsis tribuloides (Sm.) A.DC. & Fagaceae & & $\checkmark$ & & Least Concern (LC) \\
\hline 15 & Cotoneaster frigidus Wall. ex Lindl. & Rosaceae & & & $\checkmark$ & Least Concern (LC) \\
\hline 16 & Daphniphyllum himalense (Benth.) Mull. Arg. & Daphniphyllaceae & $\checkmark$ & $\checkmark$ & $\checkmark$ & Least Concern (LC) \\
\hline 17 & Deutzia staminea $\mathrm{R}$. Br. ex Wall. & Hydrangeaceae & $\checkmark$ & & & Least Concern (LC) \\
\hline 18 & Dodecadenia grandiflora Nees & Lauraceae & $\checkmark$ & $\checkmark$ & & Least Concern (LC) \\
\hline 19 & Elaeagnus parvifolia Wall. ex Royle & Elaeagnaceae & $\checkmark$ & $\checkmark$ & $\checkmark$ & Least Concern (LC) \\
\hline 20 & Eriobotrya dubia (Lindl.) Decne. & Rosaceae & & $\checkmark$ & $\checkmark$ & Data Deficient (DD) \\
\hline 21 & Eriobotrya elliptica Lindl. & Rosaceae & & & $\checkmark$ & Near Threatened (NT) \\
\hline 22 & Euonymus pendulus Wall. & Celestraceae & & $\checkmark$ & $\checkmark$ & Near Threatened (NT) \\
\hline 23 & Eurya acuminate DC. & Pentaphylacaceae & $\checkmark$ & $\checkmark$ & $\checkmark$ & Least Concern (LC) \\
\hline 24 & Euryacer asifolia (D.Don) Kobuski & Pentaphylacaceae & $\checkmark$ & $\checkmark$ & & Least Concern (LC) \\
\hline 25 & Ficus neriifolia Sm. & Moraceae & & $\checkmark$ & $\checkmark$ & Least Concern (LC) \\
\hline 26 & Ficus pumila $L$. & Moraceae & $\checkmark$ & & & Data Deficient (DD) \\
\hline 27 & Fraxinus floribund Wall. & Oleaceae & $\checkmark$ & & & Least Concern (LC) \\
\hline 28 & Garuga pinnata Roxb. & Burseraceae & & $\checkmark$ & & Data Deficient (DD) \\
\hline 29 & Hydrangea heteromalla D.Don & Hydrangeaceae & & & $\checkmark$ & Least Concern (LC) \\
\hline 30 & Ilex dipyrena Wall. & Aquifoliaceae & $\checkmark$ & $\checkmark$ & $\checkmark$ & Least Concern (LC) \\
\hline 31 & Juglans regia $L$. & Juglandaceae & $\checkmark$ & & & Least Concern (LC) \\
\hline 32 & Leucosceptrum canum Sm. & Lamiaceae & & & $\checkmark$ & Least Concern (LC) \\
\hline 33 & Ligustrum confusum Decne. & Oleaceae & $\checkmark$ & $\checkmark$ & & Data Deficient (DD) \\
\hline 34 & Lindera pulcherrima (Nees) Hook. f. & Lauraceae & $\checkmark$ & $\checkmark$ & $\checkmark$ & Least Concern (LC) \\
\hline 35 & Litsea doshia (D.Don) Kosterm. & Lauraceae & & $\checkmark$ & $\checkmark$ & Near Threatened (NT) \\
\hline 36 & Lyonia ovalifolia (Wall.) Drude & Ericaceae & $\checkmark$ & $\checkmark$ & $\checkmark$ & Least Concern (LC) \\
\hline 37 & Lyonia villosa (Hook. f.) Hand.-Mazz. & Ericaceae & & $\checkmark$ & & Least Concern (LC) \\
\hline 38 & Macaranga pustulata King ex Hook. f. & Euphorbiaceae & $\checkmark$ & & & Least Concern (LC) \\
\hline 39 & Machilus clarkeana King ex Hook. f. & Lauraceae & $\checkmark$ & & & Data Deficient (DD) \\
\hline 40 & Machilus duthiei King ex Hook. f. & Lauraceae & $\checkmark$ & $\checkmark$ & $\checkmark$ & Least Concern (LC) \\
\hline 41 & Machilus odoratissima Nees & Lauraceae & $\checkmark$ & $\checkmark$ & & Least Concern (LC) \\
\hline 42 & Magnolia doltsopa (Buch.-Ham. ex DC.) Figlar & Magnoliaceae & $\checkmark$ & $\checkmark$ & & Data Deficient (DD) \\
\hline 43 & Maytenus rufa (Wall.) H. Hara & Celastraceae & & $\checkmark$ & & Least Concern (LC) \\
\hline 44 & Myrica esculenta Buch.-Ham. ex D.Don & Myricaceae & $\checkmark$ & $\checkmark$ & $\checkmark$ & Least Concern (LC) \\
\hline 45 & Myrsine semiserrata Wall. & Primulaceae & $\checkmark$ & $\checkmark$ & $\checkmark$ & Least Concern (LC) \\
\hline 46 & Neolitsea pallens (D.Don) Momiy. \& H. Hara ex H. Hara & Lauraceae & $\checkmark$ & $\checkmark$ & $\checkmark$ & Least Concern (LC) \\
\hline 47 & Osmanthus fragrans Lour. & Oleaceae & $\checkmark$ & & & Data Deficient (DD) \\
\hline 48 & Photinia integrifolia Lindl. & Rosaceae & & $\checkmark$ & & Least Concern (LC) \\
\hline
\end{tabular}




\begin{tabular}{|c|c|c|c|c|c|c|}
\hline 49 & Pieris formosa (Wall.) D.Don & Ericaceae & $\checkmark$ & $\checkmark$ & $\checkmark$ & Least Concern (LC) \\
\hline 50 & Pinus roxburghii Sarg. & Pinaceae & $\checkmark$ & $\checkmark$ & & Least Concern (LC) \\
\hline 51 & Pinus wallichiana A.B. Jacks. & Pinaceae & & $\checkmark$ & & Least Concern (LC) \\
\hline 52 & Prunus cerasoides D.Don & Rosaceae & $\checkmark$ & $\checkmark$ & $\checkmark$ & Least Concern (LC) \\
\hline 53 & Prunus cornuta (Wall. ex Royle) Steud. & Rosaceae & $\checkmark$ & & & Least Concern (LC) \\
\hline 54 & Prunus napaulensis (Ser.) Steud. & Rosaceae & $\checkmark$ & & & Data Deficient (DD) \\
\hline 55 & Prunus rufa Hook. f. & Rosaceae & & $\checkmark$ & & Least Concern (LC) \\
\hline 56 & Prunus undulata Buch.-Ham. ex D.Don & Rosaceae & $\checkmark$ & & & Least Concern (LC) \\
\hline 57 & Pyrularia edulis (Wall. ex Roxb.) DC. & Santalaceae & $\checkmark$ & & $\checkmark$ & Least Concern (LC) \\
\hline 58 & Pyrus pashia Buch.-Ham. ex D.Don & Rosaceae & $\checkmark$ & & $\checkmark$ & Least Concern (LC) \\
\hline 59 & Quercus glauca Thunb. & Fagaceae & & $\checkmark$ & $\checkmark$ & Least Concern (LC) \\
\hline 60 & Quercus lamellosa Sm. & Fagaceae & $\checkmark$ & $\checkmark$ & $\checkmark$ & Data Deficient (DD) \\
\hline 61 & Quercus lanata Sm. & Fagaceae & & $\checkmark$ & $\checkmark$ & Least Concern (LC) \\
\hline 62 & Quercus semecarpifolia Sm. & Fagaceae & $\checkmark$ & $\checkmark$ & $\checkmark$ & Least Concern (LC) \\
\hline 63 & Rhamnus purpureus Edgew. & Rhamnaceae & $\checkmark$ & & & Least Concern (LC) \\
\hline 64 & Rhododendron arboretum Sm. & Ericaceae & $\checkmark$ & $\checkmark$ & $\checkmark$ & Least Concern (LC) \\
\hline 65 & Rhododendron barbatum Wall. ex G. Don & Ericaceae & $\checkmark$ & & & Least Concern (LC) \\
\hline 66 & Rhododendron campanulatum D.Don & Ericaceae & $\checkmark$ & & & Least Concern (LC) \\
\hline 67 & Rhus javanica Miller & Anacardiaceae & & & $\checkmark$ & Least Concern (LC) \\
\hline 68 & Rhus succedanea L. & Anacardiaceae & $\checkmark$ & $\checkmark$ & & Least Concern (LC) \\
\hline 69 & Salix obscura Andersson & Salicaceae & $\checkmark$ & & & Data Deficient (DD) \\
\hline 70 & Saurauia napaulensis DC. & Actinidiaceae & & $\checkmark$ & & Least Concern (LC) \\
\hline 71 & Schima wallichii (DC.) Korth. & Theaceae & & $\checkmark$ & & Least Concern (LC) \\
\hline 72 & Skimmia arborescens T. Anderson ex Gamble & Rutaceae & & $\checkmark$ & & Least Concern (LC) \\
\hline 73 & Sorbus vestita (Wall. ex G.Don) Lodd. & Rosaceae & $\checkmark$ & & $\checkmark$ & Least Concern (LC) \\
\hline 74 & Symplocos ramosissima Wall. ex G.Don & Symplocaceae & $\checkmark$ & $\checkmark$ & $\checkmark$ & Least Concern (LC) \\
\hline 75 & Symplocos theifolia D.Don & Symplocaceae & $\checkmark$ & $\checkmark$ & & Least Concern (LC) \\
\hline 76 & Taxu swallichiana Zucc. & Taxaceae & $\checkmark$ & & & Endangered (EN) \\
\hline 77 & Tsuga dumosa (D.Don) Eichler & Pinaceae & $\checkmark$ & & & Least Concern (LC) \\
\hline 78 & Viburnum erubescens Wall. ex DC. & Adoxaceae & $\checkmark$ & $\checkmark$ & $\checkmark$ & Least Concern (LC) \\
\hline 79 & Viburnum grandiflorum Wall. ex DC. & Adoxaceae & & & $\checkmark$ & Least Concern (LC) \\
\hline 80 & Zizyphus incurva Roxb. & Rhamnaceae & & $\checkmark$ & $\checkmark$ & Least Concern (LC) \\
\hline \multicolumn{7}{|c|}{ SHRUBS/BUSHES } \\
\hline 1 & Eleutherococcus cissifolius (Griff. ex Seem.) Harms & Araliaceae & $\checkmark$ & $\checkmark$ & & Least Concern (LC) \\
\hline 2 & Arundinaria maling Gamble & Poaceae & $\checkmark$ & $\checkmark$ & & Data Deficient (DD) \\
\hline 3 & Berberis aristata DC. & Berberidaceae & $\checkmark$ & $\checkmark$ & $\checkmark$ & Least Concern (LC) \\
\hline 4 & Berberis asiatica Roxb. ex DC. & Berberidaceae & $\checkmark$ & $\checkmark$ & & Least Concern (LC) \\
\hline 5 & Berberis insignis Hook. f. \& Thomson & Berberidaceae & & $\checkmark$ & & Least Concern (LC) \\
\hline 6 & Berberis napaulensis (DC.) Laferr. & Berberidaceae & $\checkmark$ & $\checkmark$ & $\checkmark$ & Least Concern (LC) \\
\hline 7 & Berberis wallichiana DC. & Berberidaceae & $\checkmark$ & & & Least Concern (LC) \\
\hline 8 & Boenninghausenia albiflora (Hook.) Rchb. ex Meisn. & Rutaceae & $\checkmark$ & $\checkmark$ & & Least Concern (LC) \\
\hline 9 & Colebrookea oppositifolia Sm. & Lamiaceae & & $\checkmark$ & & Least Concern (LC) \\
\hline 10 & Colquhounia coccinea Wall. & Lamiaceae & & $\checkmark$ & & Least Concern (LC) \\
\hline 11 & Cotoneaster acuminatus Lindl. & Rosaceae & $\checkmark$ & $\checkmark$ & & Least Concern (LC) \\
\hline 12 & Cotoneaster microphyllus Wall. ex Lindl. & Rosaceae & $\checkmark$ & $\checkmark$ & & Least Concern (LC) \\
\hline 13 & Daphne bholua Buch.-Ham. ex D.Don & Thymelaeaceae & $\checkmark$ & $\checkmark$ & $\checkmark$ & Near threatened (NT) \\
\hline 14 & Daphne papyracea Wall. ex Steud. & Thymelaeaceae & $\checkmark$ & $\checkmark$ & & Near threatened (NT) \\
\hline 15 & Desmodium elegans DC. & Leguminosae & & $\checkmark$ & & Least Concern (LC) \\
\hline 16 & Desmodium multiflorum DC. & Leguminosae & & $\checkmark$ & & Least Concern (LC) \\
\hline 17 & Drepanostachyum falcatum (Nees) Keng f. & Poaceae & $\checkmark$ & & & Data Deficient (DD) \\
\hline 18 & Edgeworthia gardneri (Wall.) Meisn. & Thymelaeaceae & & & $\checkmark$ & Near threatened (NT) \\
\hline 19 & Gaultheria fragrantissima Wall. & Ericaceae & & $\checkmark$ & $\checkmark$ & Least Concern (LC) \\
\hline 20 & Hypericum cordifolium Choisy & Hypericaceae & & $\checkmark$ & & Vulnerable (VU) \\
\hline 21 & Hypericum hookeranum Wight \& Arn. & Hypericaceae & $\checkmark$ & $\checkmark$ & $\checkmark$ & Least Concern (LC) \\
\hline 22 & Indigofera heterantha Wall. ex Brandis & Leguminosae & & $\checkmark$ & $\checkmark$ & Least Concern (LC) \\
\hline
\end{tabular}




\begin{tabular}{|c|c|c|c|c|c|c|}
\hline 23 & Inula cappa (Buch.-Ham. ex D.Don) DC. & Compositae & & $\checkmark$ & & Least Concern (LC) \\
\hline 24 & Lonicera ligustrina Wall. & Caprifoliaceae & $\checkmark$ & & & Data Deficient (DD) \\
\hline 25 & Maesa chisia Buch.-Ham. ex D.Don & Primulaceae & $\checkmark$ & & & Least Concern (LC) \\
\hline 26 & Mussa endatreutleri Stapf & Rubiaceae & $\checkmark$ & & & Least Concern (LC) \\
\hline 27 & Neillia rubiflora D.Don & Rosaceae & $\checkmark$ & & & Least Concern (LC) \\
\hline 28 & Phyllanthus clarkei Hook. f. & Euphorbiaceae & & $\checkmark$ & $\checkmark$ & Least Concern (LC) \\
\hline 29 & Piptanthus nepalensis (Hook.) D.Don & Leguminosae & $\checkmark$ & & & Least Concern (LC) \\
\hline 30 & Prinsepia utilis Royle & Rosaceae & $\checkmark$ & & & Least Concern (LC) \\
\hline 31 & Randia tetrasperma (Roxb.) Benth. \& Hook. f. ex Brandis & Rubiaceae & $\checkmark$ & $\checkmark$ & & Least Concern (LC) \\
\hline 32 & Ribesacum inatum Wall. ex G. Don & Grossulariaceae & $\checkmark$ & & & Least Concern (LC) \\
\hline 33 & Rosa brunonii Lindl. & Rosaceae & $\checkmark$ & & $\checkmark$ & Least Concern (LC) \\
\hline 34 & Rosa macrophylla Lindl. & Rosaceae & $\checkmark$ & & & Least Concern (LC) \\
\hline 35 & Rosa sericea Lindl. & Rosaceae & & & $\checkmark$ & Least Concern (LC) \\
\hline 36 & Rubus calycinus Wall. ex D.Don & Rosaceae & & & & Least Concern (LC) \\
\hline 37 & Rubus ellipticus Sm. & Rosaceae & $\checkmark$ & $\checkmark$ & $\checkmark$ & Data Deficient (DD) \\
\hline 38 & Rubus pentagonus Wall. ex Focke & Rosaceae & $\checkmark$ & & & Least Concern (LC) \\
\hline 39 & Rubus sumatranus Miq. & Rosaceae & & $\checkmark$ & & Data Deficient (DD) \\
\hline 40 & Sarcococca saligna (D.Don) Mull. Arg. & Buxaceae & $\checkmark$ & $\checkmark$ & & Data Deficient (DD) \\
\hline 41 & Sarcococca wallichii Stapf & Buxaceae & $\checkmark$ & $\checkmark$ & & Least Concern (LC) \\
\hline 42 & Spiraea canescens D.Don & Rosaceae & & & $\checkmark$ & Data Deficient (DD) \\
\hline 43 & Swidao blonga (Wall.) Sojak & Cornaceae & $\checkmark$ & & & Least Concern (LC) \\
\hline 44 & Viburnum cylindricum Buch.-Ham. ex D.Don & Sambucaceae & & $\checkmark$ & $\checkmark$ & Least Concern (LC) \\
\hline 45 & Viburnum mullaha Buch.-Ham. ex D.Don & Sambucaceae & & $\checkmark$ & $\checkmark$ & Least Concern (LC) \\
\hline 46 & Wikstroemia canescens Meisn. & Thymelaeaceae & & $\checkmark$ & & Least Concern (LC) \\
\hline 47 & Zanthoxylum armatum DC. & Rutaceae & $\checkmark$ & $\checkmark$ & $\checkmark$ & Least Concern (LC) \\
\hline 48 & Zanthoxylum oxyphyllum Edgew. & Rutaceae & $\checkmark$ & $\checkmark$ & $\checkmark$ & Least Concern (LC) \\
\hline \multicolumn{7}{|c|}{ WOODY CLIMBERS } \\
\hline 1 & Ampelocissus rugosa (Wall.) Planch. & Vitaceae & & $\checkmark$ & & Least Concern (LC) \\
\hline 2 & Aristolochia griffithii Hook. f. \& Thoms. Ex Duch. & Aristolochiaceae & $\checkmark$ & $\checkmark$ & $\checkmark$ & Least Concern (LC) \\
\hline 3 & Ceropegia longifolia Wall. & Apocyanaceae & $\checkmark$ & $\checkmark$ & $\checkmark$ & Data Deficient (DD) \\
\hline 4 & Cissampelos pareira $L$. & Menispermaceae & & & $\checkmark$ & Least Concern (LC) \\
\hline 5 & Clematis connata DC. & Ranunculaceae & $\checkmark$ & & & Least Concern (LC) \\
\hline 6 & Clematis montana Buch.-Ham. ex DC. & Ranunculaceae & $\checkmark$ & & & Least Concern (LC) \\
\hline 7 & Cochlianthus gracilis Benth. & Leguminoceae & $\checkmark$ & & & Data Deficient (DD) \\
\hline 8 & Euonymus echinatus Wall. & Celastraceae & $\checkmark$ & $\checkmark$ & & Least Concern (LC) \\
\hline 9 & Hedera nepalensis K. Koch & Araliaceae & $\checkmark$ & $\checkmark$ & $\checkmark$ & Least Concern (LC) \\
\hline 10 & Hedyotis scandens Roxb. & Rubiaceae & & $\checkmark$ & & Data Deficient (DD) \\
\hline 11 & Holboellia latifolia Wall. & Lardizabalaceae & $\checkmark$ & $\checkmark$ & $\checkmark$ & Least Concern (LC) \\
\hline 12 & Hoya edenii King ex Hook. f. & Apocyanaceae & $\checkmark$ & $\checkmark$ & & Endangered (EN) \\
\hline 13 & Jasminum humile L. & Oleaceae & $\checkmark$ & $\checkmark$ & & Least Concern (LC) \\
\hline 14 & Jasminum officinale L. & Oleaceae & $\checkmark$ & $\checkmark$ & & Least Concern (LC) \\
\hline 15 & Piper mullesua Buch.-Ham. ex D.Don & Piperaceae & $\checkmark$ & $\checkmark$ & & Least Concern (LC) \\
\hline 16 & Rubia manjith Roxb. ex Fleming & Rubiaceae & $\checkmark$ & $\checkmark$ & $\checkmark$ & Least Concern (LC) \\
\hline 17 & Rubus acuminatus Sm. & Rosaceae & & $\checkmark$ & & Least Concern (LC) \\
\hline 18 & Rubus paniculatus Sm. & Rosaceae & $\checkmark$ & $\checkmark$ & $\checkmark$ & Least Concern (LC) \\
\hline 19 & Sabia campanulata Wall. ex Roxb. & Sabiaceae & $\checkmark$ & $\checkmark$ & & Least Concern (LC) \\
\hline 20 & Schisandra grandiflora (Wall.) Hook. f. \& Thomson & Schisandraceae & $\checkmark$ & & & Least Concern (LC) \\
\hline 21 & Smilax aspera $L$. & Smilacaceae & $\checkmark$ & & $\checkmark$ & Least Concern (LC) \\
\hline 22 & Smilax elegans Wall. ex Kunth & Smilacaceae & & $\checkmark$ & $\checkmark$ & Least Concern (LC) \\
\hline 23 & Smilax ferox Wall. ex Kunth & Smilacaceae & $\checkmark$ & $\checkmark$ & $\checkmark$ & Least Concern (LC) \\
\hline 24 & Smilax menispermoidea A. DC. & Smilacaceae & $\checkmark$ & & & Least Concern (LC) \\
\hline 25 & Tetrastigma serrulatum (Roxb.) Planch. & Vitaceae & $\checkmark$ & $\checkmark$ & $\checkmark$ & Least Concern (LC) \\
\hline
\end{tabular}


Annex II: Distribution maps of the species recorded from the three Mid-hills regions of Nepal, based on the herbarium specimens deposited at the RBGE, BM and KATH
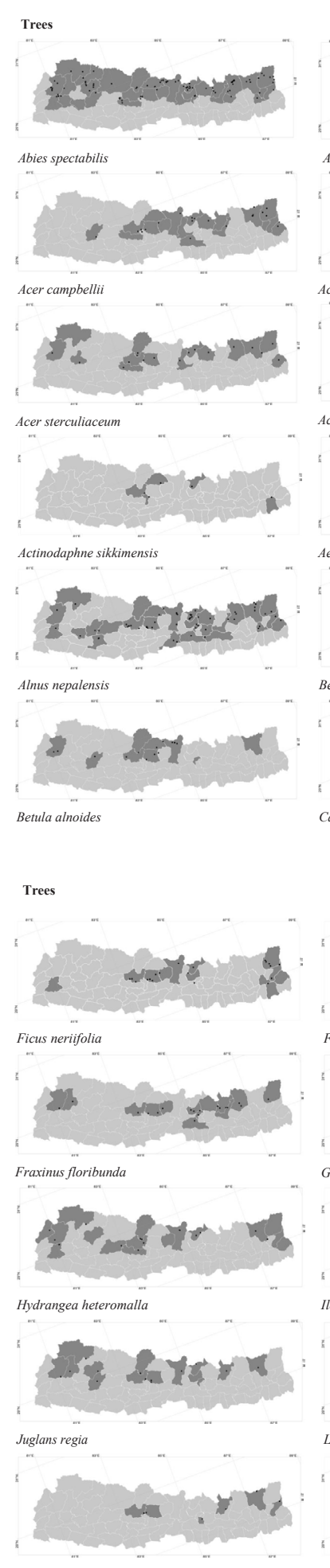

Ligustrum confusum

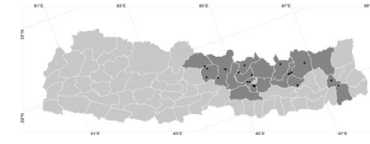

Litsea doshia
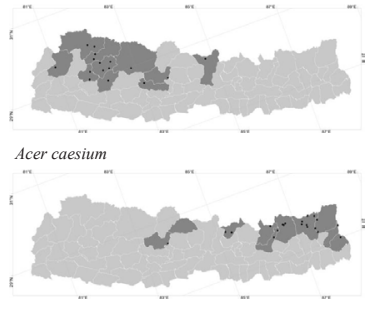

Acer caudatum

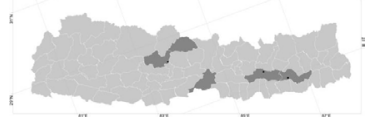

Actinodaphne angustifolia

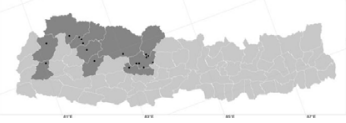

\section{Aesculus indica}

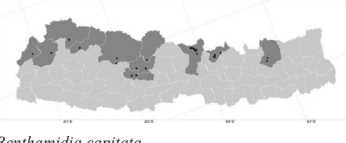

Benthamidia capitata
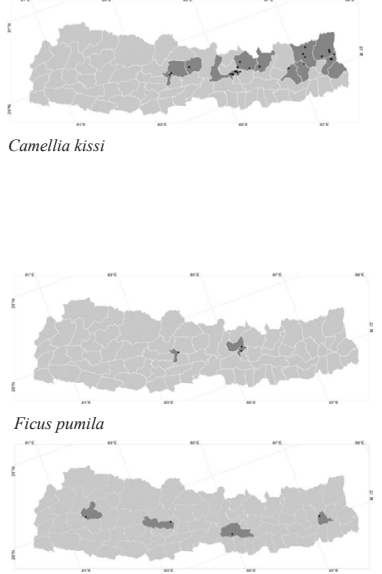
Garuga pinnata

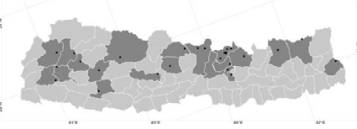
Ilex dipyrena

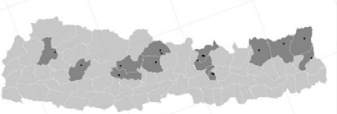

Leucosceptrum canum

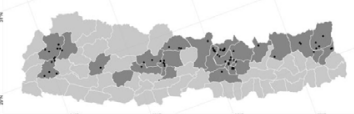

Lindera pulcherrima

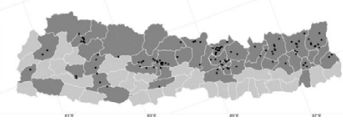

Lyonia ovalifolic
Trees
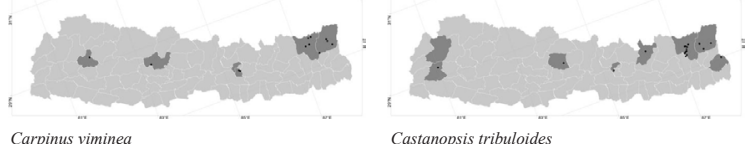

$\underset{\substack{\text { Carpinus viminea } \\ \mathrm{nt}}}{\mathrm{n}}$
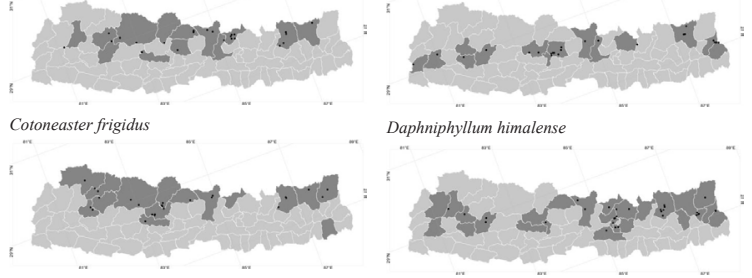

Daphniphyllum himalense

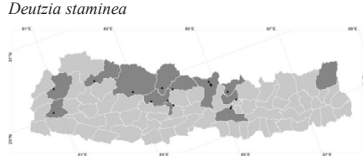

Elaeagnus parvifolia
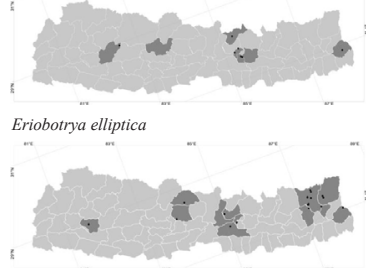

Eurya acuminata

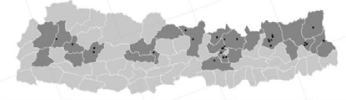

Dodecadenia

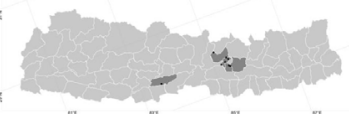

Eriobotrya dub
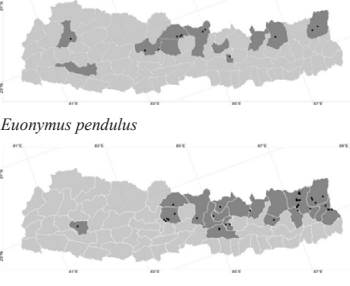

Trees

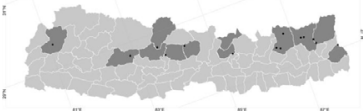

Lyoni avillosa

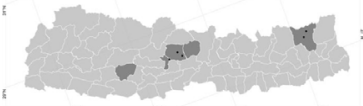

Machilus clarkeana

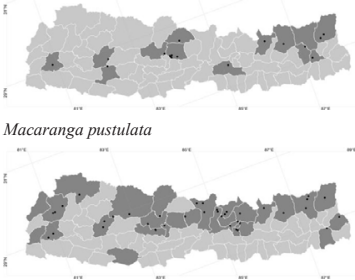

Machilus duthiei

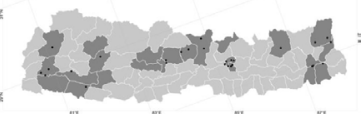

Machilus odoratissima

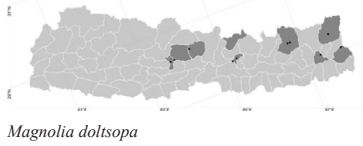

Magnolia doltsopa

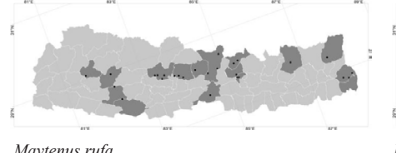

t3 andy ?

Maytenus rufa

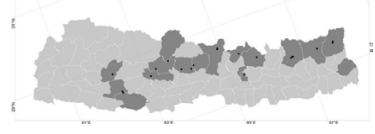

Myrsinesemis errata

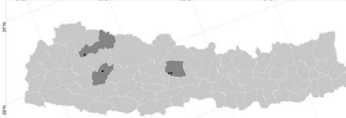

Osmanthus fragran
Myrica esculenta

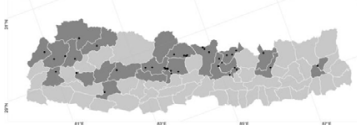

Neolitsea pallens

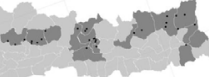




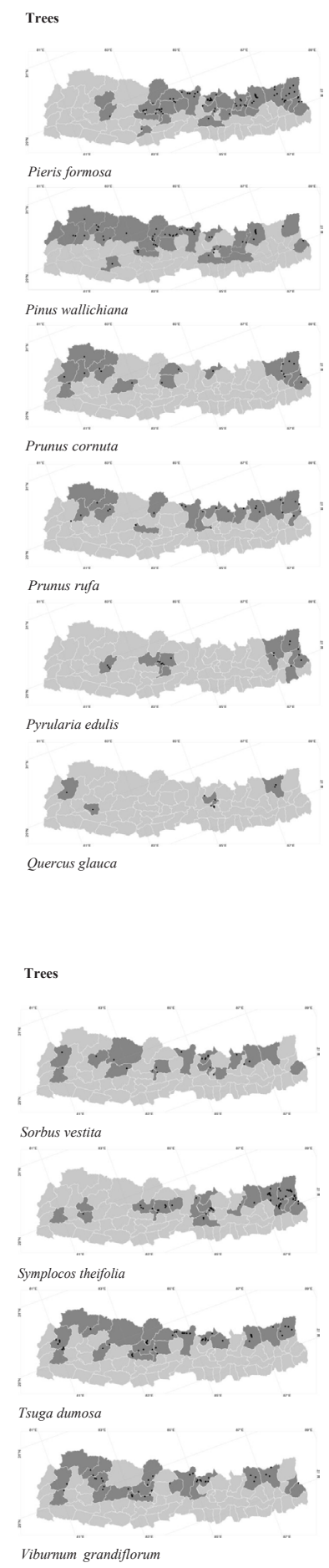

Trees

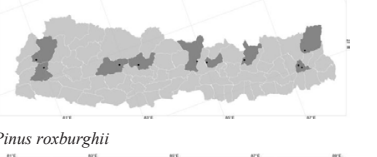

$+2+48$ Prunus cerasoides

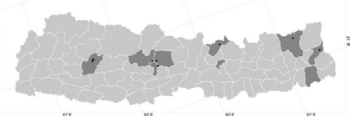
Prunus napaulensis
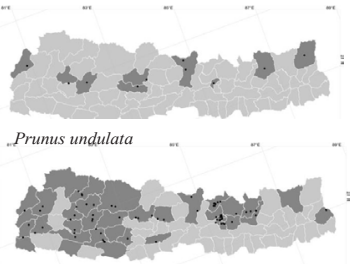
Pyrus pashia

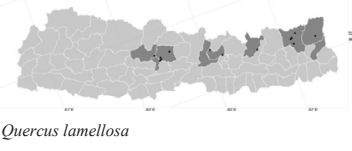

Quercus lamellosa
$1+204$ Quercus lanata

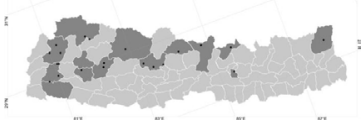
Rhamnus purpureus

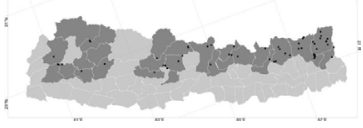
Rhododendron barbatum

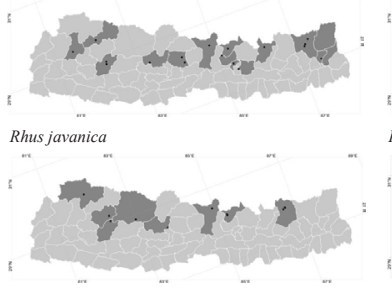
Salix obscura
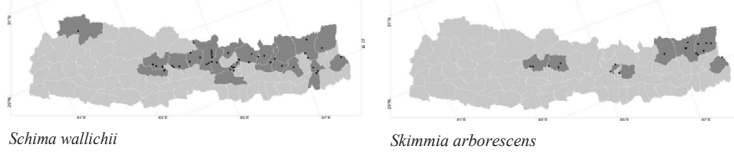

Shrubs
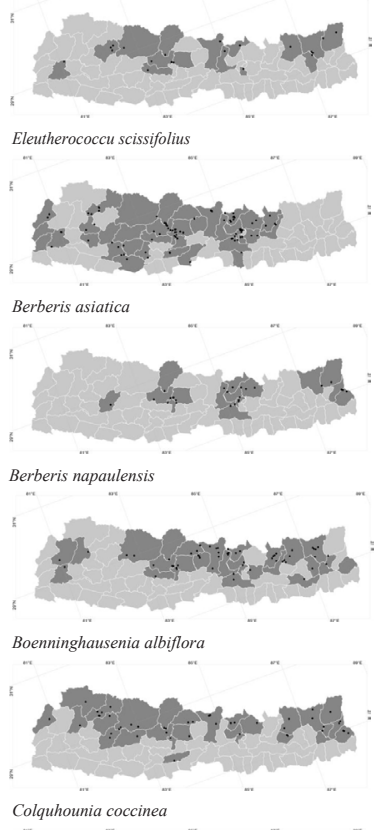

tondowent
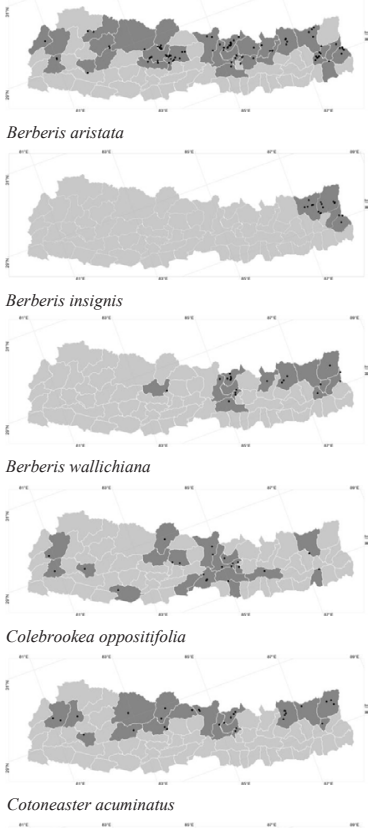

$4+2.2+23+2$ 


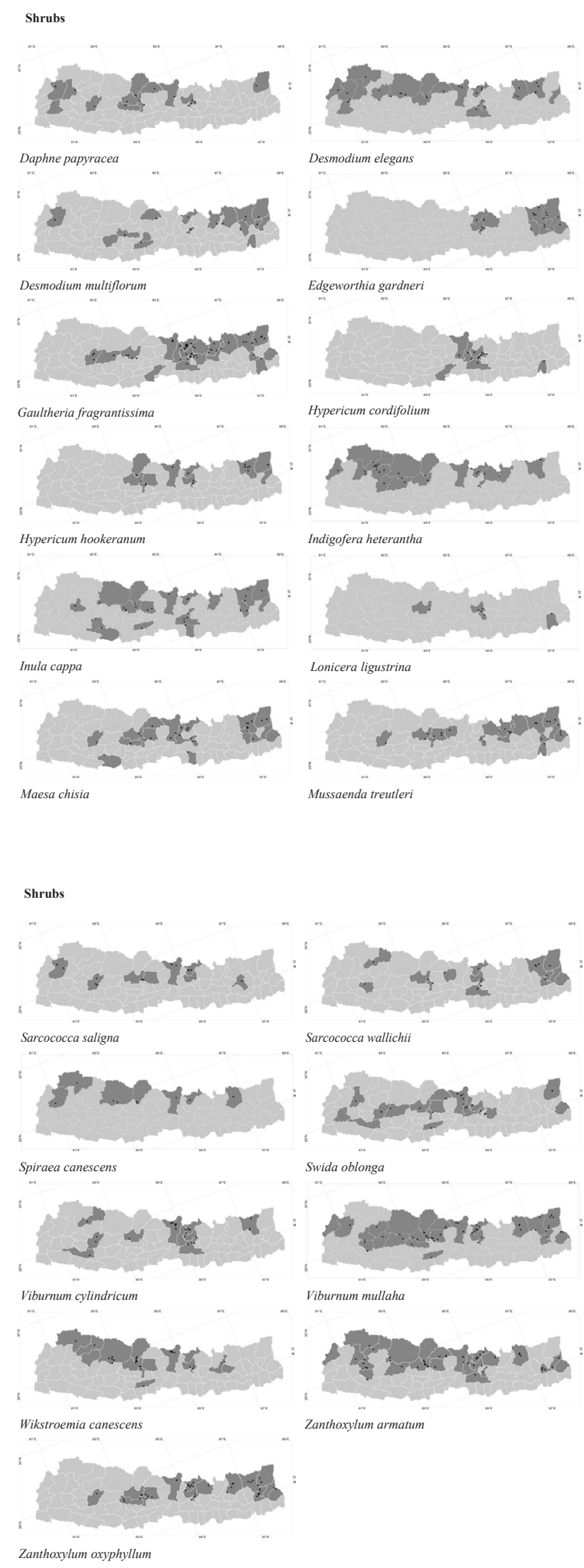

Shrubs
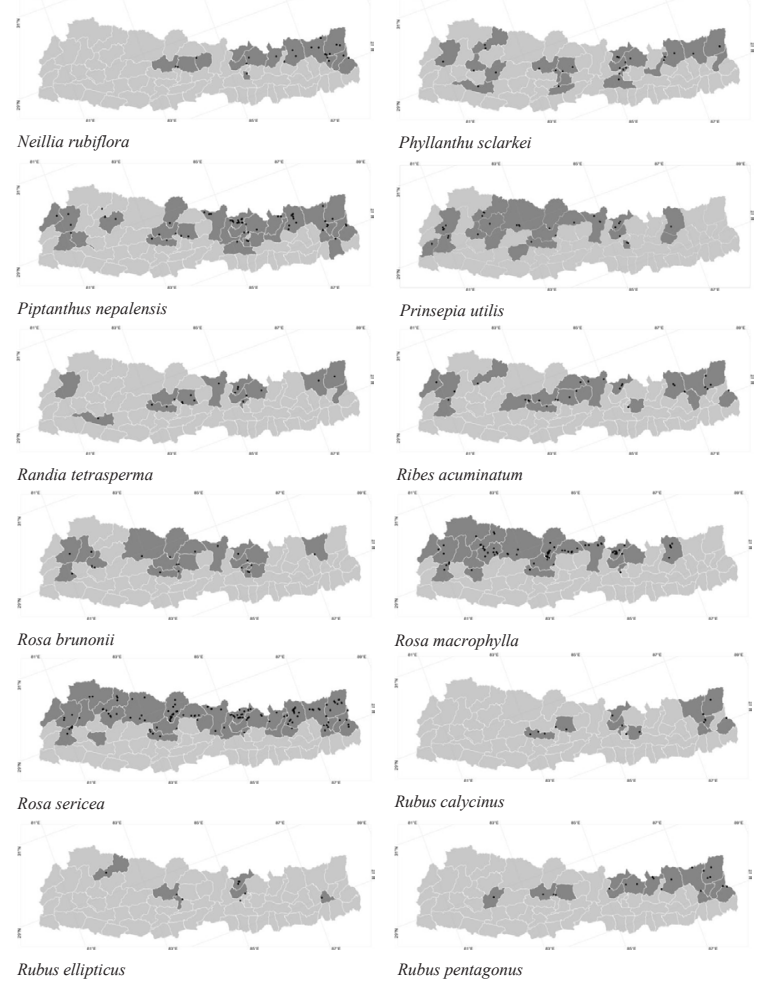

Climbers
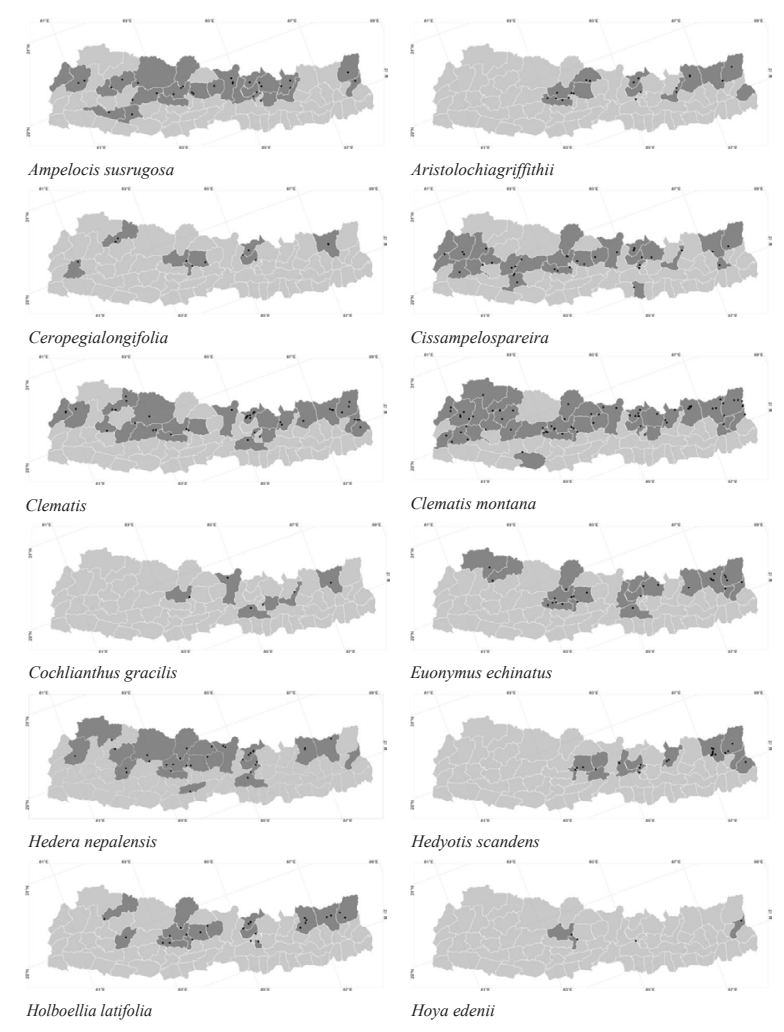


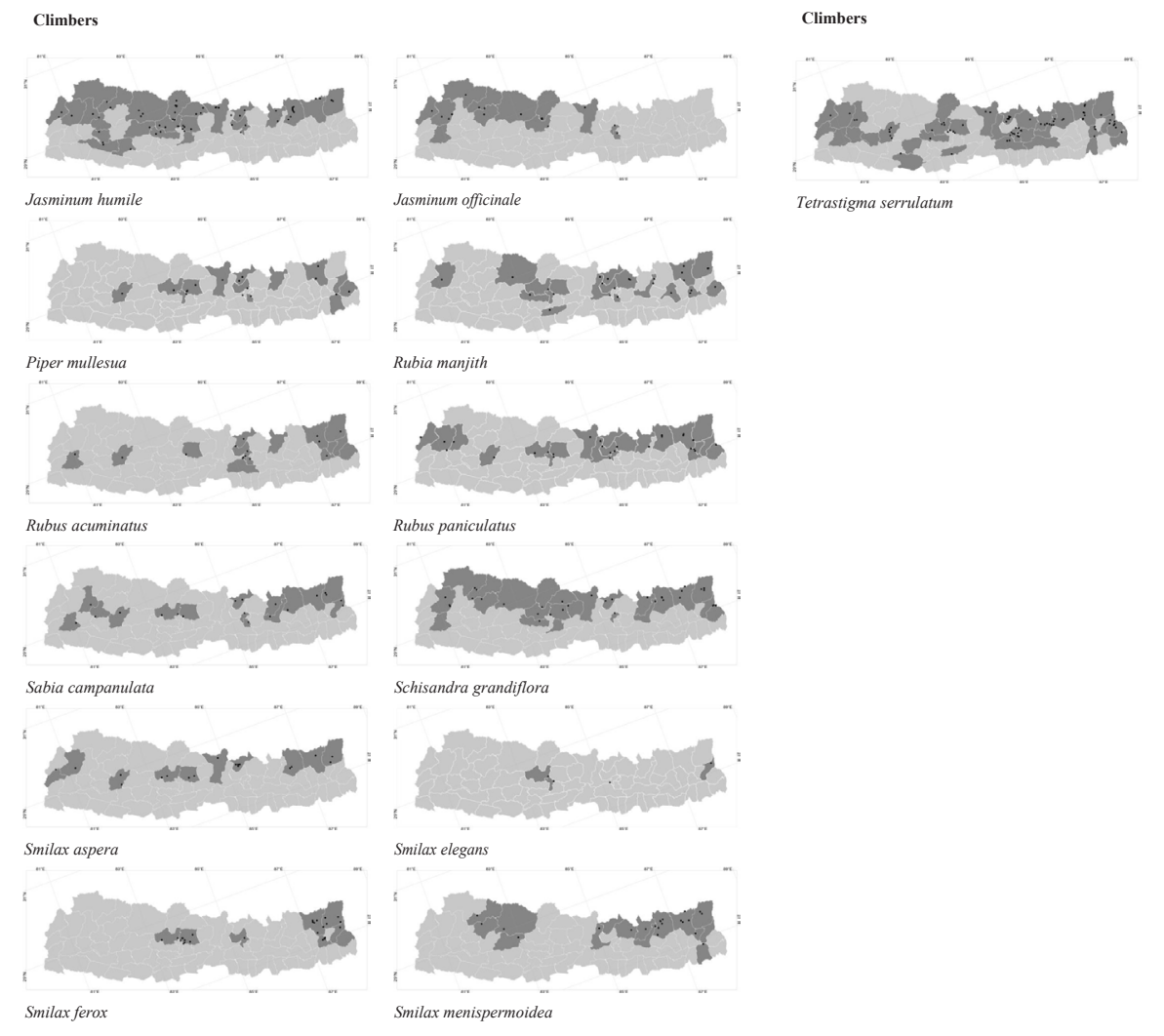

